\title{
Windkessel model of hemodynamic state supported by a pulsatile ventricular assist device in premature ventricle contraction
}

\author{
Keun Her', Joon Yeong Kim², Ki Moo $\mathrm{Lim}^{3}$ and Seong Wook Choi²*
}

\author{
${ }^{*}$ Correspondence: \\ swchoe@kangwon.ac.kr \\ ${ }^{2}$ Program of Mechanical \\ and Biomedical Engineering, \\ College of Engineering, \\ Kangwon National University, \\ Chuncheon-si, Republic \\ of Korea \\ Full list of author information \\ is available at the end of the \\ article
}

\begin{abstract}
Background: Counter-pulsation control (CPC) by ventricular assist devices (VADs) is believed to reduce cardiac load and increase coronary perfusion. However, patients with VADs have a higher risk of arrhythmia, which may cause the CPC to fail. Consequently, CPC has not been applied by VADs in clinical practice. The phase-locked loop (PLL) algorithm for CPC is readily implemented in VADs; however, it requires a normal, consistent heartbeat for adequate performance. When an arrhythmia occurs, the algorithm maintains a constant pumping rate despite the unstable heartbeat. Therefore, to apply the PLL algorithm to CPC, the hemodynamic effects of abnormal heartbeats must be analyzed.
\end{abstract}

Objectives: This study sought to predict the hemodynamic effects in patients undergoing CPC using VADs, based on electrocardiogram (ECG) data, including a wide range of heart rate (HR) changes caused by premature ventricular contraction (PVC) or other reasons.

Methods: A four-element Windkessel hemodynamic model was used to reproduce the patient's aortic blood pressure in this study. ECG data from 15 patients with severe congestive heart failure were used to assess the effect of the CPC on the patients' hemodynamic state. The input and output flow characteristics of the pulsatile VAD (LibraHeart I, Cervika, Korea) were measured using an ultrasound blood flow meter (TS410, Transonic, USA), with the aortic pressure maintained at 80-120 mmHg. All other patient conditions were also reproduced.

Results: In patients with PVCs or normal heartbeats, CPC controlled by a VAD reduced the cardiac load by 20 and $40 \%$, respectively. When the HR was greater for other reasons, such as sinus tachycardia, simultaneous ejection from the heart and VAD was observed; however, the cardiac load was not increased by rapid cardiac contractions resulting from decreased left ventricle volume. These data suggest that the PLL algorithm reduces the cardiac load and maintains consistent hemodynamic changes.

Keywords: Windkessel model, Pulsatile ventricular assist device, Counter-pulsation control, Phase-locked loop, Arrhythmia

\section{Background}

Use of a ventricular assist device (VAD) is the most effective way to improve the survival rates of patients with end-stage heart failure [1-5]. However, to avoid additional heart 
problems or thrombus, new approaches such as pulsatile flow and counter-pulsation control (CPC) are required [5, 6]. Although CPC is expected to increase cardiovascular circulation and reduce cardiac load, it has not been implemented clinically because of the possible adverse effects on the heart in the event of CPC failure [7-9]. Patients with VADs are at a higher risk of developing arrhythmia, which can induce CPC failure [10, 11]. Therefore, prior to engaging in CPC, it should be confirmed that failure of the VAD will not cause the patient's hemodynamic state or cardiac load to worsen.

Multiple studies of CPC using VADs have assumed normal HRs without considering the possible occurrence of arrhythmias. Lim et al. reported that CPC increased coronary perfusion and decreased cardiac load [7]. Koshimoto et al. assessed such factors using pulsatile rotary VADs in animal studies [8,9]. Some CPC algorithms have considered tachycardia and bradycardia; however, this approach has not been tested clinically or in animals due to the difficulties associated with inducing arrhythmias [12]. Clinical studies involving CPC are complicated despite the increasing use of VAD applications. This is because many reports have suggested that VADs should not be used for patients with arrhythmias [13-15]. Moreover, most VADs used to date did not have a CPC function $[12,16]$. Intra-aortic balloon pumps (IABPs) have successfully engaged in CPC and beneficially affected heart treatment $[17,18]$. However, in cases involving VADs, it is difficult to apply the CPC algorithm for IABPs. IABPs inject helium gas through a catheter into the balloon in the aorta rapidly, whereas the outflow pulse of a VAD as it passes through the blood stream is slow $[9,18,19]$. Therefore, the CPC algorithm of VADs should predict future heartbeats based on prior heartbeats, and preemptively provide subsequent pulses [12].

The LibraHeart I (Cervika, Korea) is a pulsatile VAD that analyzes prior heart beats and initiates its next pulse flow to keep the time interval between the pulses of the patient's heart and the VAD constant according to the phase locked loop (PLL) algorithm [6]. The PLL is an algorithm that determines the pumping rate of a VAD whose output pulses are related to the QRS complex of the patient's electrocardiogram (ECG) [20]. However, the PLL algorithm for a VAD can fail at CPC when arrhythmias occur. Therefore, the likelihood of CPC failure, and the effects it would have on cardiac load and the hemodynamic state, should be investigated before determining whether to implement CPC using VADs. This study analyzed ECG data from 15 patients with congestive heart failure and observed 35 arrhythmias, including premature ventricular contractions (PVCs) and sinus tachycardia (ST), in those ECGs [21].

The hemodynamic model was based on the four-element Windkessel model, which reproduces patient blood pressure and blood flow data [22, 23]. Because the conventional Windkessel model does not consider the reflection wave in the aorta, the actual blood pressure and blood flow data of a patient cannot be reproduced [24]; whereas the four-element model considers the reflection wave and reproduces the same aortic pressure and blood flow as measured in the patient [25]. The four-element model has been used to evaluate the accuracy of medical devices that analyze blood pressure and blood flow [22].

The purpose of this study was to predict the effects of a VAD in CPC mode on the cardiac and hemodynamic states using a four-element Windkessel model that includes the data for a VAD and arrhythmias that induced CPC failure. 


\section{Methods}

\section{Counter pulsation control by the ventricular assist device using the phase-locked loop method}

The conventional method of determining when to initiate VADs following heartbeat detection misses the appropriate counter-pulsation point. If the delay from the time of heart rate (HR) detection to actuator operation is not reduced to within $0.1 \mathrm{~s}$ [9], a normal heartbeat of over $70 \mathrm{bpm}$ can be missed. Following heartbeat detection, delay occurs due to the time required to establish the pulse flow by increasing the actuator speed, thereby generating the blood pressure pulse at the outlet of the VAD and delivering the pulse to the aortic valve through vessels and blood [18]. Comparatively, an IABP successfully applies CPC and reduces delays by injecting helium gas through a stiff, narrow catheter to a balloon located near the heart [19]. To overcome the slow response of a pulsatile VAD, the controller should predict the starting point by analyzing the regularity of the heartbeat and increasing motor speed prior to detecting the following beat [6, 12].

A pulsatile VAD (LibraHeart I, Cervika, Korea) uses a PLL algorithm to determine the starting point for pulse generation (Fig. 1) [6, 26]. The VAD controller sets the pumping rate (PR) of the VAD to equal the patient's HR. The HR is determined by measuring the heartbeat time $\left(T_{p}\right)$ and calculating the beating period $\left(T_{R-R}\right)$. The heartbeat time $\left(T_{p}\right)$ is determined from ECG data using the Tompkins method [27]. The HR is calculated as the average reciprocal of the normal $\mathrm{T}_{\mathrm{R}-\mathrm{R}}$ over a 10 -s period. The means and standard deviations of all $T_{R-R}$ values during the 10 -s period are calculated, and $T_{R-R}$ values that fall within the range of the mean and standard deviation are regarded as normal values. Since arrhythmias, including PVC, affect the $T_{R-R}$ average and $H R$, if the PR is

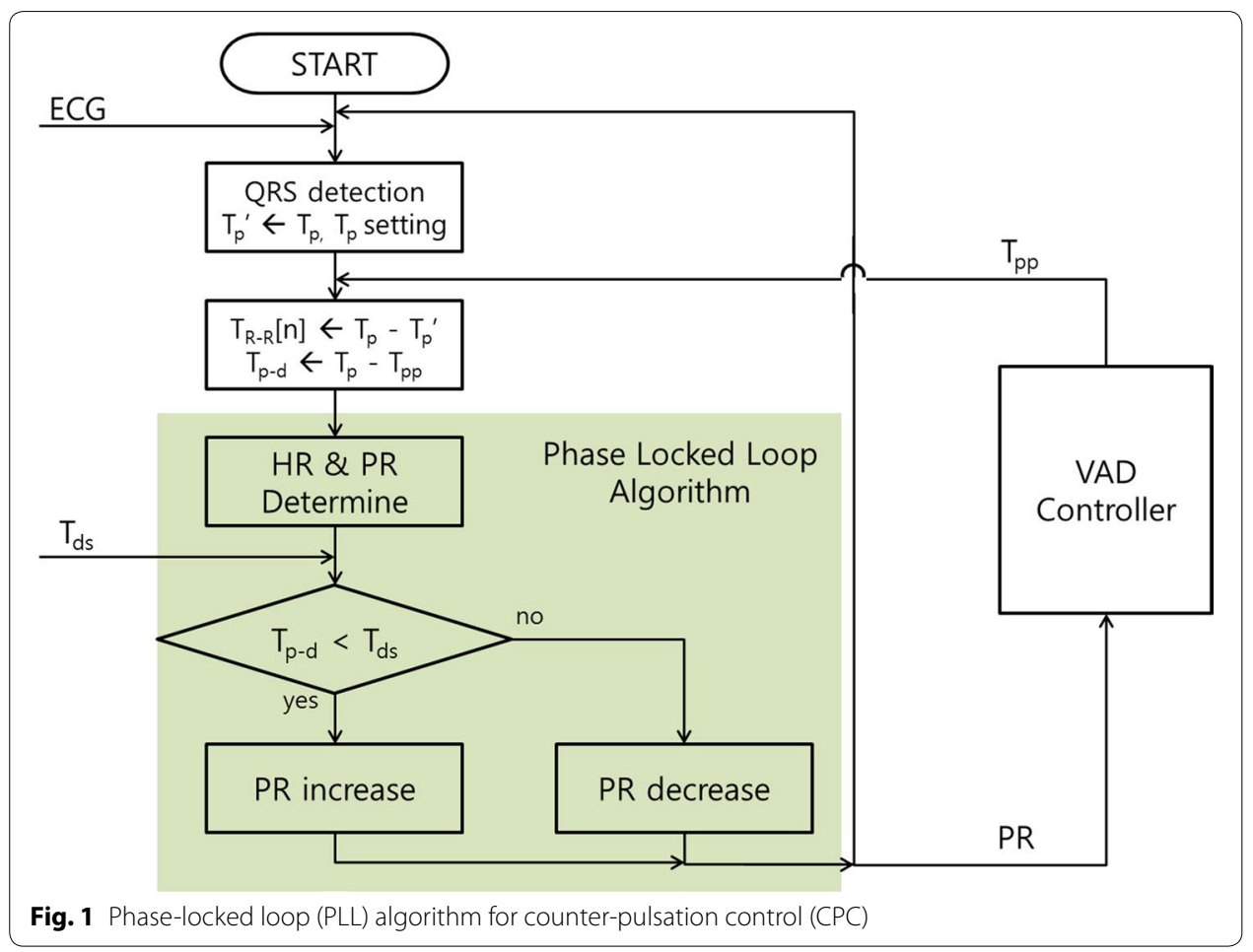


affected by outlying data, the CPC of a VAD may have constant errors for even normal heartbeats. As the onset of pulse generation is determined prior to the heartbeat being detected, the delay $\left(\mathrm{T}_{\mathrm{p}-\mathrm{d}}\right)$ between the heartbeat and the VAD pulse is calculated immediately following $T_{p}$ detection. When the $T_{p-d}$ differs from the predetermined optimal value $\left(\mathrm{T}_{\mathrm{ds}}\right)$, the ensuing VAD pulse is adjusted temporarily to bring $\mathrm{T}_{\mathrm{ds}}$ to within $5 \%$ of the average $T_{R-R}$. $T_{d s}$ was determined such that the ratio of $T_{p-d}$ to $T_{R-R}$ was $45 \%$.

When an arrhythmia occurred, $\mathrm{T}_{\mathrm{R}-\mathrm{R}}$ changed temporarily from 30 to $170 \%$, which caused temporary CPC failure. In most cases, however, the VAD slowly adjusted its PR to the average HR according to the PLL algorithm, and it seemed to maintain a constant PR as opposed to changing the PR of the IABP for each heartbeat.

\section{Hemodynamic model of the left ventricle and aorta}

The hemodynamic changes were investigated using the four-element Windkessel model. The conventional Windkessel model shows the relationship of diastolic and systolic blood pressure and blood flow [24]. However, it does not reproduce the actual blood pressure and blood flow waveform, so that a more sophisticated model, such as a fourelement Windkessel model, is necessary [22, 25]. The four elements of the Windkessel model are the aortic compliance, aortic impedance (resistance and inductance of the aorta), resistance of the peripheral arteries, and compliance of the peripheral arteries. These elements are necessary to reproduce the reflection wave, actual blood pressure, and blood flow information of an actual patient [25]. The values used in previous studies were used again to reproduce the blood pressure and blood flow data, as shown in Table 1 [22].

To compare the characteristics of the cardiac load in the four-element Windkessel model and to analyze the effect of the VAD, the compliance of the LV, the resistance of the mitral and aortic valves, and the LA pressure were determined, as shown in Table 2.

The $\mathrm{C}_{\mathrm{LV}}$ is the ratio of volume to pressure in the $\mathrm{LV}$ when the internal pressure changes from 5 to $120 \mathrm{mmHg}$ and the blood volume changes from 65 to $120 \mathrm{cc}$ (Fig. 2c) [24]. The values of $R_{A V}$ and $R_{M V}$ are the resistance of valve opening and closing according to the direction of the blood flow [24]. $\mathrm{P}_{\mathrm{LA}}$ is the pressure of the left atrium and was determined to be $10 \mathrm{mmHg}$, which is the normal left atrium pressure.

In the absence of the VAD, HRs of $70 \mathrm{bpm}$ resulted in an arterial pressure of $80-120 \mathrm{mmHg}$ and an average cardiac output of $4 \mathrm{~L} / \mathrm{min}$, as shown in Fig. 2b. With the exception of the VAD connection, the Windkessel model was based on patient data, with the reflection wave being adjusted to reproduce the same waveform as for the patient's actual aortic blood pressure (AoP) $[22,23]$. When a pulsatile VAD that implements the

Table 1 Parameters related to reflection wave in the 4-element Windkessel model

\begin{tabular}{llll}
\hline Symbol & Values & Units & Meaning of parameter \\
\hline$C_{\text {Aorta }}$ & 0.15 & $\mathrm{cc} / \mathrm{mmHg}$ & Compliance of the aorta \\
$Z_{\text {Aorta }}$ & & & \\
$L_{\text {Aorta }}$ & 0.0015 & $\mathrm{cc} / \mathrm{s}^{2}$ & Impedance of the aorta composed of inertia and resistance of blood flow \\
$R_{\text {Aorta }}$ & 0.08 & $\mathrm{mmHg} \mathrm{s} / \mathrm{cc}$ & \\
$C_{\text {PA }}$ & 1.5 & $\mathrm{cc} / \mathrm{mmHg}$ & Compliance of the peripheral arteries \\
$\mathrm{R}_{\text {PA }}$ & 1.35 & $\mathrm{mmHg} \mathrm{s} / \mathrm{cc}$ & Resistance of the peripheral vessels \\
\hline
\end{tabular}


Table 2 Auxiliary parameters used to estimate the cardiac load supported by the VAD

\begin{tabular}{|c|c|c|c|}
\hline Symbol & Values & Units & Meaning of parameter \\
\hline \multirow[t]{2}{*}{$C_{L V}$} & 12.5 & $\mathrm{cc} / \mathrm{mmHg}$ & Compliance of the LV during LV relaxation \\
\hline & $0.66-12.5$ & & Compliance of the LV during LV contraction \\
\hline \multirow[t]{2}{*}{$\mathrm{R}_{\mathrm{AV}}$} & 0.002 & $\mathrm{mmHg} s / c \mathrm{c}$ & Resistance of the aortic valve when it opens \\
\hline & 1000 & & Resistance of the aortic valve when it closes \\
\hline \multirow[t]{2}{*}{$\mathrm{R}_{\mathrm{MV}}$} & 0.01 & $\mathrm{mmHg} s / c \mathrm{c}$ & Resistance of the mitral valve when it opens \\
\hline & 1000 & & Resistance of the mitral valve when it closes \\
\hline$P_{L A}$ & 10 & $\mathrm{mmHg}$ & Blood pressure in the left atrium \\
\hline VAD & 2 (average) & $\mathrm{L} / \mathrm{min}$ & Blood flow through the VAD \\
\hline
\end{tabular}

a
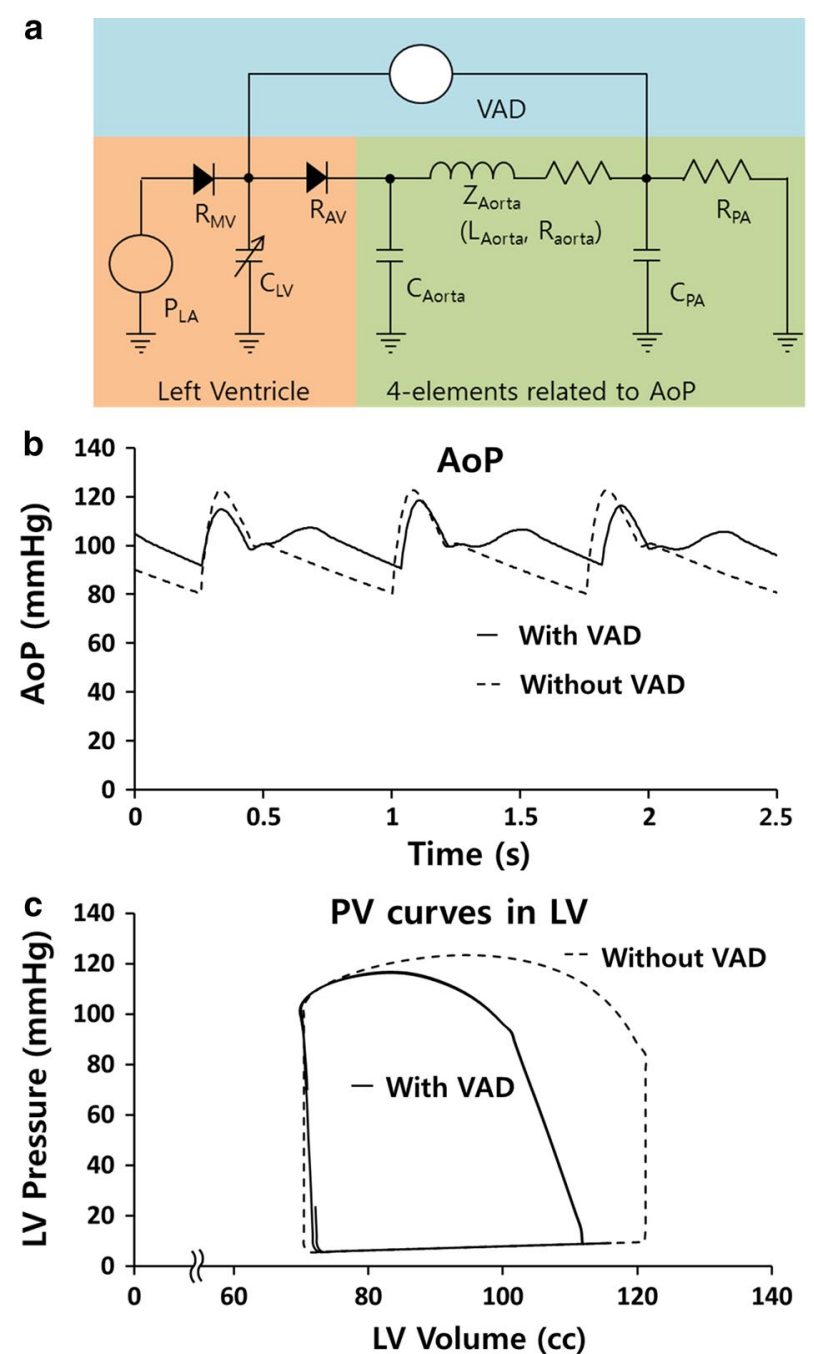

Fig. 2 a 4-element Windkessel model, including the ventricular assist device (VAD), b aortic blood pressure $(A \circ P)$, and $\mathbf{c}$ pressure-volume curves before and during VAD perfusion

PLL algorithm is applied to the Windkessel model, the systolic (diastolic) BP should decrease (increase). This should reduce the LV volume, and the decrease in LV output and LV pressure will result in a decrease in load, which is similar to the results of 
previous animal experiments [6]. The Windkessel model initiated LV contractions at the QRS wave on the ECG, as shown in Fig. 3. As a result, when an arrhythmia occurred on the ECG, the changes in HR induced changes in the blood pressure and blood flow in the LV and the aorta of the Windkessel model.

ECG data were obtained from 15 patients ( 11 men, aged $22-71$ years; 4 women, aged 54-63 years) with severe congestive heart failure (NYHA class 3-4) at a sampling rate of 250 per second. The data included 1260 heart beats and 28 arrhythmias [21]. Nine patients had tachycardia with a rapid HR over $100 \mathrm{bpm}$. The primary arrhythmias were PVCs (Fig. 3a), which animal studies suggest are more likely to occur when using a pulsatile VAD [6]. Although the contraction force of the left ventricle was set to be constant, the Windkessel model predicted that the AoP would decrease during PVC (Fig. 3b), as shown in animal studies. In total, 25 PVCs were analyzed among 15 patients and were found to increase the HR by $15.5-34.5 \%$. LV contractions following a PVC occurred regularly, so the $\mathrm{T}_{\mathrm{R}-\mathrm{R}}$ following PVCs was temporarily extended, changing the hemodynamic states in the model. Such hemodynamic changes at PVC and at the next beat were observed and compared as the LV stroke volume fluctuated. The HR changes caused by PVCs were applied to two Windkessel models, one with a VAD and one without a VAD. Three episodes of ST occurred in two patients and resulted in simultaneous ejection of the heart and VAD. The effect of co-pulsations was compared with the results for normal counter-pulsation. In addition, the relationship between temporary heart rate changes and the cardiac load was investigated by applying HR changes over a wide range to the model.

The inflow and outflow data for the VAD (LibraHeart I, Cervika, Korea) were measured using an ultrasonic blood flow meter (TS410, Transonic, USA) using a mock circulation system that reproduces the patient's hemodynamic conditions [26]. As shown in Fig. 4a, the mock circulation system consists of a compliance chamber that maintains the afterload of the VAD from 80 to $100 \mathrm{mmHg}$ and an open chamber that maintains the preload of the VAD at $10 \mathrm{mmHg}$ as the AoP of the Windkessel model and the patient.
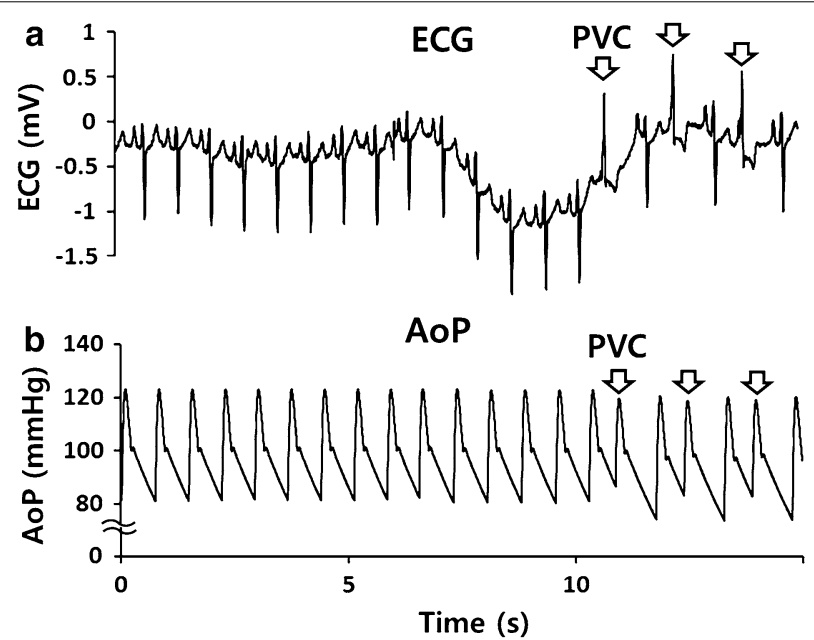

Fig. 3 a Representative electrocardiogram (ECG) data showing temporary premature ventricle contraction (PVC) obtained from a congestive heart failure patient, and $\mathbf{b}$ the patient's AoP, reproduced by the Windkessel model 
a
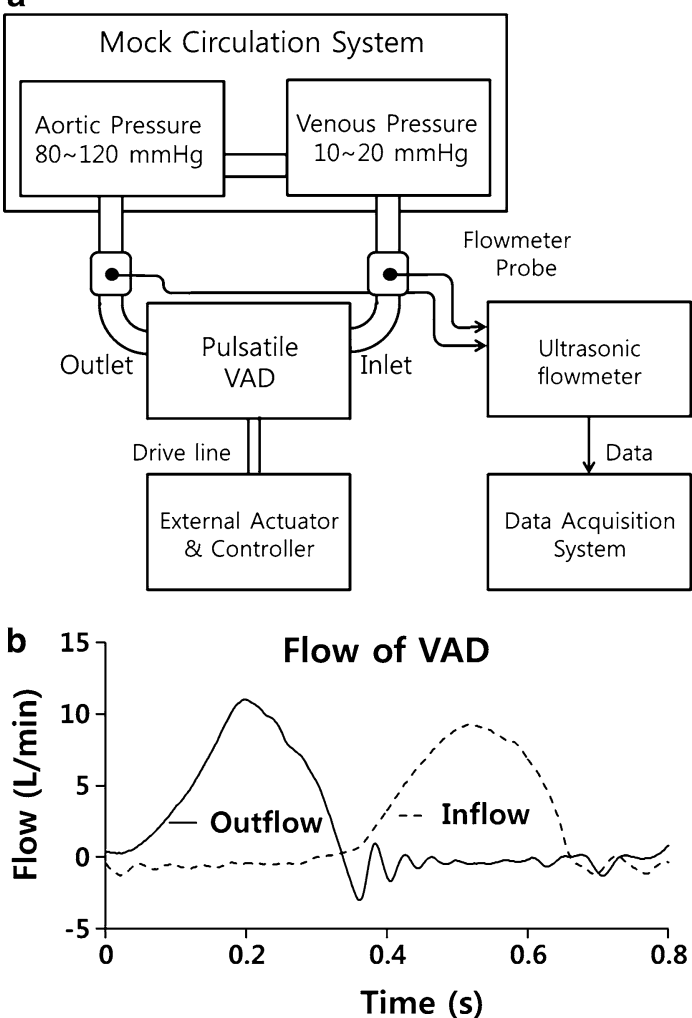

Fig. 4 a Experimental setup for the flow measurement of the pulsatile $V A D$, and $\mathbf{b}$ the inflow and outflow waveform of the VAD

The pulse volume of the VAD was fixed at $29 \mathrm{cc}$, so that the outflow of the VAD reached $2 \mathrm{~L} / \mathrm{min}$, which is $50 \%$ of the normal body perfusion rate when the pulse rate was $70 \mathrm{bpm}$ without a VAD (Fig. 4b). The model assumes that the inlet and outlet of the VAD are connected to the LV and the descending aorta, as shown in Fig. 2a.

\section{Results}

PVCs stopped the blood flow from the left atrium to the LV and reduced the maximum volume within the ventricle during a heartbeat, as shown in Fig. 5. In Fig. 5, (1) and (2) are representative of normal ventricular contractions before PVCs, while (3) is representative of a case when a PVC occurs. The heartbeat immediately following the PVC occurred at the normal time point, but the volume of the ventricle increased beyond the normal value due to the increased time between the PVC and the subsequent heartbeat (Fig. 5d). The PV curve in Fig. 5d shows the changes in ventricular pressure and volume caused by PVCs. Changes in the area of the closed curve reveal that the cardiac load differed between PVC and normal heartbeats.

The volume of the ventricle was reduced by the inflow to the VAD (Fig. 5e). When PVC occurred and the ratio of $T_{P-D} / T_{R-R}$ deviated significantly from the target value (45\%), the cardiac output and the output of the VAD did not occur simultaneously, because the blood flow output period of the VAD was too short, so the VAD output finished before the start of the following PVC. As shown in Fig. 5b, c, the diastolic AoP increased due 

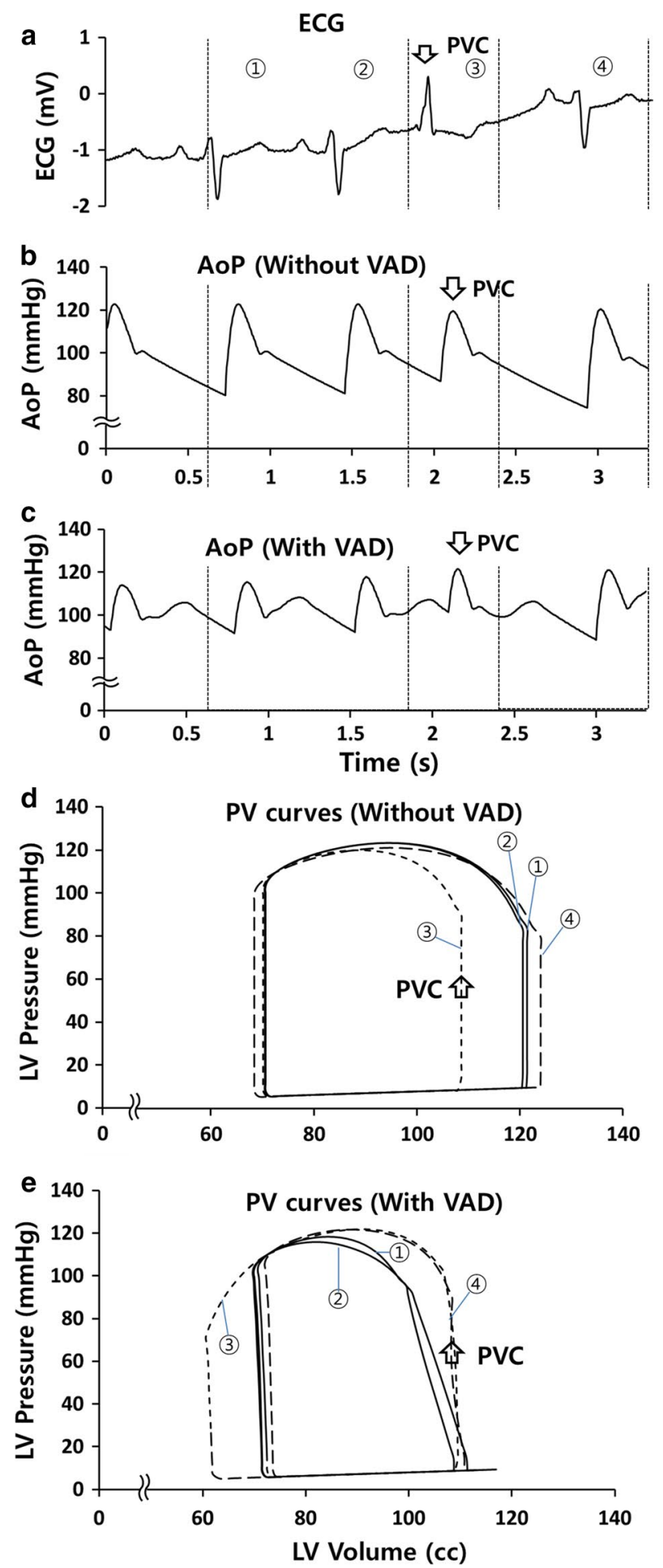

Fig. 5 Representative curves for a patient ECGs, b AoP without VAD perfusion, c AoP with VAD perfusion, d pressure-volume (PV) curves of LV without VAD, and e PV curves of LV with VAD. At first, the patient's heart beat was normal (1), (2)). However, when PVC occurred, the heart rate increased abruptly (3) and then immedicately decreased (4) 

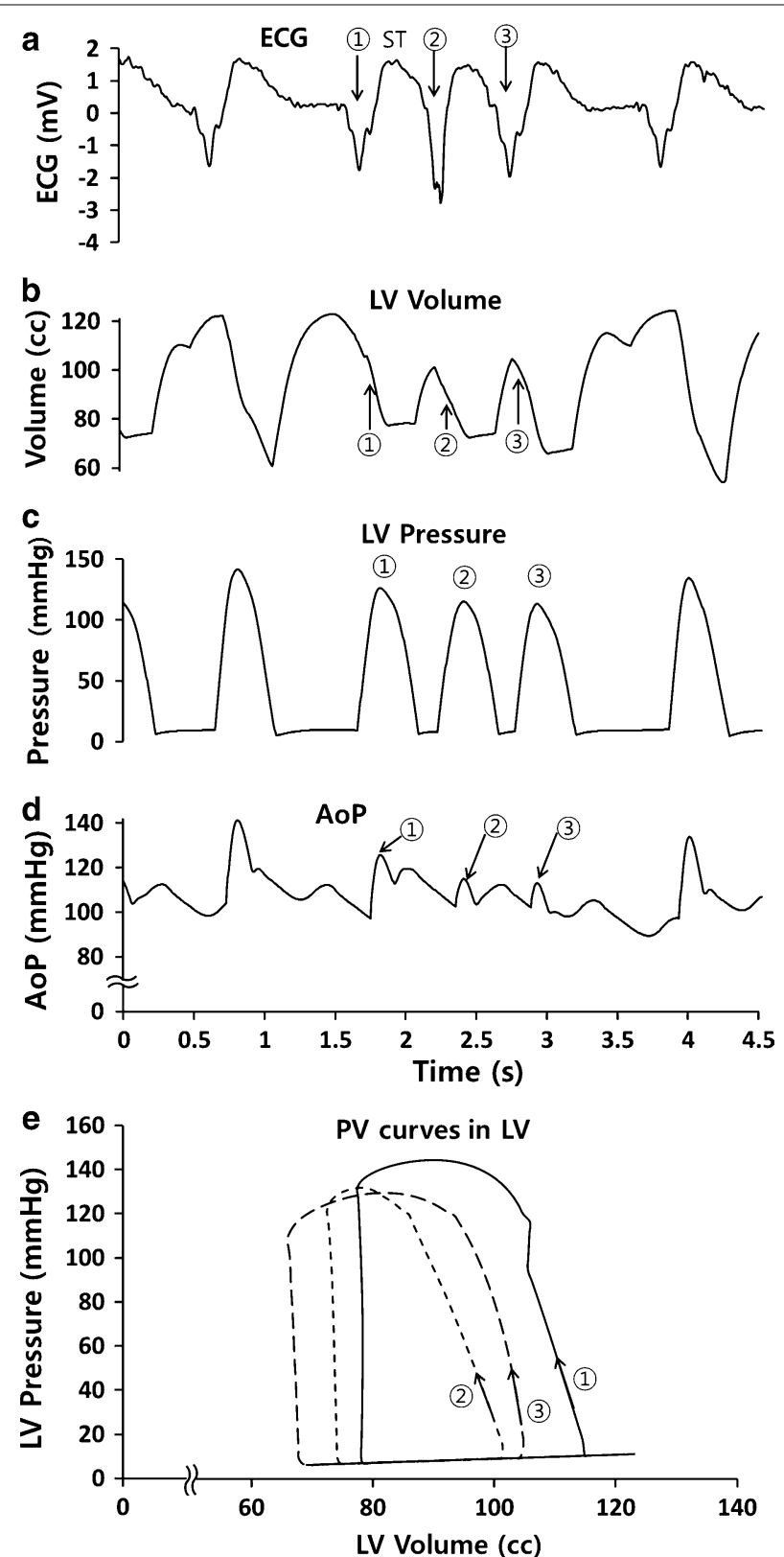

Fig. 6 Representations of a patient ECGs, b $L V$ volumes, $\mathbf{c} L V$ pressures, $\mathbf{d} A \circ P$ and $\mathbf{e} P V$ curves during normal (1) and subsequent abnormal heart beat caused by sinus tachycardia (2), (3))

to the output of the VAD, while the systolic pressure decreased due to decreases in the cardiac output caused by the flow from the LV to the VAD. The VAD pulses caused the area of the PV curve to decrease (Fig. 5e). Even when the PVC was generated, the VAD reduced the volume of the ventricle, which subsequently reverted to its prior size following the PVC.

When ST occurred, successive rapid heartbeats induced co-pulsation, as shown in Fig. 6a. However, the change in LV volume remained small because the VAD pumped blood from the LV to the aorta continuously (Fig. 6b). The systolic BP did not increase, but decreased (Fig. 6d). After finishing ST, the diastolic BP decreased slightly as the 
heart rate decreased. The PV curves showed that CPC failure did not increase the cardiac load.

As shown in Fig. 7b, a total of 1260 heart beats occurred in 15 patients, including 25 PVCs and three episodes of ST (10 beats). Considering the 1225 beats excluding PVCs and $\mathrm{ST}$, the ratio of $\mathrm{T}_{\mathrm{p}-\mathrm{d}}$ to $\mathrm{T}_{\mathrm{R}-\mathrm{R}}$ also changed from 20 to $60 \%$ due to unknown $\mathrm{HR}$ changes and tachycardia (> $100 \mathrm{bpm})$. When the ratio of $\mathrm{T}_{\mathrm{p}-\mathrm{d}}$ to $\mathrm{T}_{\mathrm{R}-\mathrm{R}}$ was within $20-60 \%$, the cardiac load was expected to decrease by $20-40 \%$ (Fig. 7a).

\section{Discussion and conclusion}

Although PVCs are not dangerous arrhythmias, their frequency affects the occurrence of dangerous arrhythmias such as ventricular fibrillation [28, 29]. PVC data enable analysis of the effect of various types of arrhythmia, because PVC shows fast and slow heart rate changes within a short period. In animal studies using pigs and a PLL-controlled VAD, CPC by the VAD induced clear changes and the systolic blood pressure decreased by $23 \%$, while the diastolic BP increased by $25 \%$ [6]; this resulted from the different characteristics of animals compared with humans and edema that was observed at thoracotomy. In vivo, PVCs occurred, when the VAD operated not in CPC mode, but in asynchronous mode. Changes in ventricular load caused by VADs are thought to cause PVCs [30]. As a result, the animal studies did not show the effects of CPC on the hemodynamic state during arrhythmias, because arrhythmias did not occur in the presence of CPC. As the causes of PVCs include alcohol consumption and stress [28], PVC can still
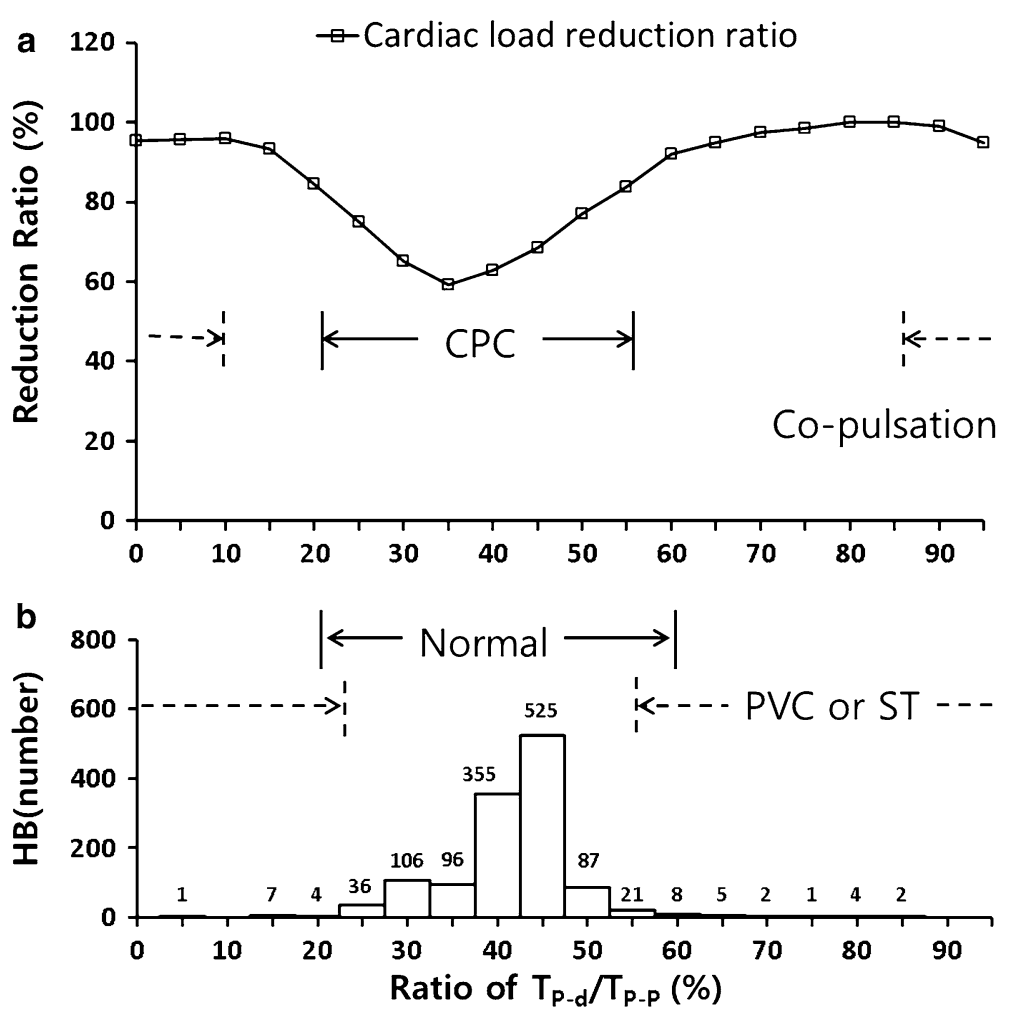

Fig. 7 a Predicted cardiac load reduction ratio according to the ratio of $T_{p-d}$ to $T_{R-R}$, and $\mathbf{b}$ histogram of the ratios of $T_{p-d}$ to $T_{R-R}$ for observed patients' heartbeats 
occur, even when a VAD imparts CPC; however, they are difficult to detect due to their intermittency. Thus, PVCs can be the prevailing arrhythmia, despite all efforts to avoid the use of VADs in arrhythmia patients.

The results of this study were obtained from simulations; however, the input and output blood flow data of the VAD were measured under the same conditions based on patients and in vitro [26]. The ECG data were measured from patients who required the application of a VAD [21]. The Windkessel model was designed using accepted mathematical and physical formulas relating to hemodynamic states [22]. The parameters in the model, including blood pressure, cardiac output, and systemic vascular resistance, were identical to those in another study and were measured in patients [22, 25]. Since the model includes the AoP waveform, which is similar to that measured in patients, the model is considered to include hemodynamic data [23]. Therefore, the results of this study are expected to be realistic for patients with similar hemodynamic characteristics.

The model used in this study predicted the effects of the VAD on events that were difficult to reproduce in clinical or animal experiments. However, a limitation of this study is that predictions using other input conditions require a substantial volume of data to be collected beforehand. Because the cardiovascular characteristics of patients vary due to multiple factors, including the influence of sympathetic and parasympathetic nervous systems and the patient's movement and posture, predictions must encompass a wide range of conditions. As the technologies for acquiring data become more encompassing, the limitations of numerical simulation will be minimized [31, 32].

Previous studies of CPC were designed to detect heart rhythms and to maintain the VAD at constant intervals $[9,12]$. However, in those studies, the VAD was expected to alter the blood flow irregularly, when irregular heartbeats occurred [33]. It is difficult to predict the outcome of CPC by a VAD under arrhythmia conditions, given that the algorithm accounts for complex scenarios to avoid co-pulsation [12, 19]. Because the Novacor left ventricular assist system (LVAS) (Novacor, Oakland, CA, USA) also has a PLL algorithm, our results should be useful for predicting the hemodynamic effects of the device during an arrhythmia [34].

In this study, the PLL algorithm showed a 97.3\% success rate at CPC when tachycardia and arrhythmias appeared in patients and it could reduce the heart load. It predicted that CPC and temporary CPC failure would not induce an increased cardiac load, even when co-pulsation occurred.

Authors' contributions

HK wrote this manuscript. KJY participated in vitro experiments. LKM did the simulation of Windkessel model. CSW analyzed obtained data. All authors read and approved the final manuscript.

Author details

${ }^{1}$ Department of Cardiovascular and Thoracic Surgery, Soonchunhyang University Hospital, Bucheon-si, Republic of Korea. ${ }^{2}$ Program of Mechanical and Biomedical Engineering, College of Engineering, Kangwon National University, Chuncheon-si, Republic of Korea. ${ }^{3}$ Department of Medical IT Convergence Engineering, Kumoh National Institute of Technology, Gumi, Republic of Korea.

\section{Acknowledgements}

This work was supported by the National Research Foundation of Korea (NRF) (C1013660-01-01) and was also supported by the Ministry of Trade, Industry \& Energy (MOTIE), Korea Institute for Advancement of Technology (KIAT) through the Encouragement Program for The Industries of Economic Cooperation Region (No. R0004491). 
Availability of data and materials

All data and material are obtained or produced through legal procedures. They have not been published elsewhere and they are not under consideration by another journal.

\section{Consent for publication}

All authors consent for the publication of this manuscript.

\section{Ethics approval and consent to participate}

Not applicable.

\section{Funding}

National Research Foundation of Korea (NRF) (C1013660-01-01). Ministry of Trade, Industry \& Energy (MOTIE), Korea Institute for Advancement of Technology (KIAT) (No. R0004491).

\section{Publisher's Note}

Springer Nature remains neutral with regard to jurisdictional claims in published maps and institutional affiliations.

Received: 21 July 2017 Accepted: 10 January 2018

Published online: 02 February 2018

\section{References}

1. Slaughter MS, Sihgn R. The role of ventricular assist devices in advanced heart failure. Rev Esp Cardiol. 2012;65(11):983-5

2. Calvaruso DF, Ocello S. Implantation of a Berlin heart as single ventricle by-pass on Fontan circulation in univentricular heart failure. ASAIO. 2007;53:e1-2.

3. Trivedi JR, Cheng A, Singh R, Williams ML, Slaughter MS. Survival on the heart transplant waiting list: impact of continuous flow left ventricular assist device as bridge to transplant. Ann Thorac Surg. 2009;98:830-4.

4. Frazier $\mathrm{OH}$, Myers TJ, Westaby S, Gregoric ID. Use of the Jarvik 2000 left ventricular assist system as a bridge to heart transplantation or as destination therapy for patients with chronic heart failure. Ann Surg. 2003;237:631-7.

5. Schmitto JD, Hanke JS, Rojas SV, Avsar M, Haverich A. First implantation in man of a new magnetically levitated left ventricular assist device. J Heart Lung Transplant. 2015;34(6):858-60.

6. Choi SW, Nam KW, Lim KM, Shim EB, Won YS, Woo HM, Kwak HH, Noh MR, Kim IY, Park SM. Effect of counter-pulsation control of a pulsatile left ventricular assist device on working load variations of the native heart. Biomed Eng Online. 2014;13:35.

7. Lim KM, Kim IS, Choi SW, Min BG, Won YS, Kim HY, Shim EB. Computational analysis of the effect of the type of LVAD flow on coronary perfusion and ventricular afterload. J Physiol Sci. 2009;59:307-16.

8. Shi Y, Korakianitis T, Bowles C. Numerical simulation of cardiovascular dynamics with different types of VAD assistance. J Biomech. 2007;40(13):2919-33.

9. Koshimoto Y, Takewa Y, Arakawa M, Umeki A, Ando M, Nishimura T, Fujii Y, Mizuno T, Nishimura M, Tatsumi E. Development of a novel drive mode to prevent aortic insufficiency during continuous-flow LVAD support by synchronizing rotational speed with heartbeat. J Artif Organs. 2013;16:129-37.

10. Rosenbaum AN, Kremers WK, Duval S, Sakaguchi S, John R, Eckman PM. Arrhythmias in patients with cardiac implantable devices after implantation of a left ventricular assist device. ASAIO. 2016;62(3):274-80.

11. Refaat M, Chemaly E, Lebeche D, Gwathmey JK, Hajjar RJ. Ventricular arrhythmias after left ventricular assist device implantation. Pacing Clin Electrophysiol. 2008;31:1246-52.

12. Nam KW, Chung J, Choi SW, Kim WE, Sun K, Ming BG. Development of counterpulsation algorithm for a movingactuator type pulsatile LVAD. Int J Artif Organs. 2004;27(10):882-90.

13. Harding JD, Piacentino V 3rd, Gauhan JP, Houser SR, Margulies KB. Electrophysiological alterations after mechanical circulatory support in patients with advanced cardiac failure. Circulation. 2001;104(11):1241-7.

14. Fasseas P, Kutalesk SP, Samuels FL, Holmes EC, Samuels LE. Ventricular assist device support for management of sustained ventricular arrhythmias. Tex Heart Inst J. 2002;29:33-6.

15. Ziv O, Dizon J, Thosni A, Naka Y, Magnano AR, Garan H. Effects of left ventricular assist device therapy on ventricular arrhythmias. J Am Coll Cardiol. 2005;45:1428-34.

16. Harding JD, Piacentino V 3rd, Rothman S, Chambers S, Jessup M, Margulies KB. Prolonged repolarization after ventricular assist device support is associated with arrhythmias in humans with congestive heart failure. J Card Fail. 2005; 11(3):227-32.

17. Annamalai SK, Buiten L, Esposito ML, Paruchuri V, Mullin A, Breton C, Redicini R, O'kelly R, Morine K, Wessler B, Patel AR, Kieman MS, Karas RH, Kapur NK. Acute hemodynamic effects of intra-aortic balloon counterpulsation pumps in advanced heart failure. J Card Fail. 2017. https://doi.org/10.1016/j.cardfail.2017.05.015.

18. Parissis H, Graham V, Lampridis S, Lau M, Hooks G, Mhandu C. IABP: history-evolution-pathophysiology-indications: what we need to know. J Cardiothorac Surg. 2016:11:122

19. Farrar DJ, Compton PG, Lawson JH, Hershon JJ, Hill JD. Control modes of a clinical ventricular assist device. IEEE Eng Med Biol Mag. 1986;5:19-24.

20. Miura H, Arai S, Sato F, Matsuki H, Sato T. A synchronous rectification using a digital PLL technique for contactless power supplies. IEE Trans Magn. 2005;41:3997-9.

21. Goldberger AL, Amaral LAN, Glass L, Hausdorff JM, Ivanov PCh, Mark RG, Mietus JE, Moody GB, Peng CK, Stanley HE. PhysioBank, PhysioToolkit, and PhysioNet: components of a new research resource for complex physiologic signals. Circulation. 2000:101(23):e215-20. 
22. Choi SW. Estimation of blood pressure diagnostic methods by using the four elements blood pressure model simulating aortic wave reflection. J Biomed Eng Res. 2015;36:183-90.

23. Linton NWF, Linton RAF. Estimation of changes in cardiac output from the arterial blood pressure waveform in the upper limb. Br J Anaesth. 2001;86:486-96.

24. Wesseling KH, Jansen JRC, Settels JJ, Schreuder JJ. Computation of aortic flow from pressure in humans using a nonlinear, three-element model. J Appl Physiol. 1993;74:2566-73.

25. Stergiopulos N, Westerh of BE, Westerhof $\mathrm{N}$. Total arterial inertance as the fourth element of the windkessel model. Am J Physiol. 1999;276:H81-8.

26. Kang SM, Choi SW. Blood flow and pressure evaluation for a pulsatile conduit-shaped ventricular assist device with structural characteristic of conduit shape. Trans Korean Soc Mech Eng B. 2009;35:1191-8.

27. Hamilton PS, Tompkins WJ. Quantitative investigation of QRS detection rules using the MIT/BIH arrhythmia database. IEEE Trans Biomed Eng. 1986;BME-33(9):1157-65.

28. Yokokawa M, Kim HM, Eric G, et al. Relation of symptoms and symptom duration to premature ventricular complex induced cardiomyopathy. Heart Rhythm. 2012;9:92-5.

29. Baman TS, Lange DC, Ilg KJ, et al. Relationship between burden of premature ventricular complexes and left ventricular function. Heart Rhythm. 2010;7:865-9.

30. Stacy G Jr, Jobe RL, Taylor LK, Hansen DE. Stretch-induced depolarizations as a trigger of arrhythmias in isolated canine left ventricles. Am J Physiol. 1992;263:613-21.

31. Wong KKL, Cheung SCP, Yang W, Tu J. Numerical simulation and experimental validation of swirling flow in spiral vortex ventricular assist device. Int J Artif Organs. 2010;33(12):856-67.

32. Wong KKL, Tu J, Kelso RM. Vortical flow analysis. J Mech Med Biol. 2010;10(02):191-212.

33. Michael RF. Mechano-electrical feedback in ventricular myocardium. Cardiovasc Res. 1996;32(15-24):30

34. Robbins RC, Kown MH, Portner PM, Oyer PE. The totally implantable novacor left ventricular assist system. Ann Thorac Surg. 2001;71:S162-5.

Submit your next manuscript to BioMed Central and we will help you at every step:

- We accept pre-submission inquiries

- Our selector tool helps you to find the most relevant journal

- We provide round the clock customer support

- Convenient online submission

- Thorough peer review

- Inclusion in PubMed and all major indexing services

- Maximum visibility for your research

Submit your manuscript at www.biomedcentral.com/submit 